\title{
An Autopsy Case of Pulmonary Aspergillosis with Fungus Ball Formation in an Artificial Aortic Graft
}

\author{
Hideaki Yamakawa ${ }^{1,2}$, Noboru Takayanagi ${ }^{1}$, Takashi Ishiguro ${ }^{1}$, Yosuke Miyahara ${ }^{1}$, \\ Yoshihiko Shimizu ${ }^{3}$ and Yutaka Sugita ${ }^{1}$
}

\begin{abstract}
A 38-year-old man with Marfan syndrome underwent an aortic replacement with an artificial aortic valve at 27 years of age and an aortic graft at 31 years of age. In 2011, he was diagnosed as having chronic necrotizing pulmonary aspergillosis (CNPA). He developed a fever and an increased sputum volume and was admitted to the hospital in 2012. Contrast-enhanced CT showed an irregularly shaped nonenhanced structure in the aortic graft. He died on hospital day 31. From the autopsy findings, we speculated that an infiltration by the CNPA lesion into a pulmonary vein was followed by the hematogenous formation of a fungus ball in the aortic graft.
\end{abstract}

Key words: chronic necrotizing pulmonary aspergillosis, disseminated aspergillosis, Marfan syndrome, graft infection

(Intern Med 52: 2117-2119, 2013)

(DOI: 10.2169/internalmedicine.52.0211)

\section{Introduction}

Chronic necrotizing pulmonary aspergillosis (CNPA) does not exhibit obviously invasive growth except under special pathological conditions such as a decreased ability to defend against infection. Cases of CNPA that progress to systemic dissemination are rare. We herein report our experience with the rare case of a patient with Marfan syndrome who developed CNPA followed by the formation of a fungus ball in his artificial aortic graft that resulted in disseminated aspergillosis.

\section{Case Report}

The patient was a 38-year-old Japanese man who had been diagnosed with Marfan syndrome. He underwent an aortic replacement with an artificial aortic valve at 27 years of age and an aortic graft 31 years of age. A computed tomography (CT) scan in 2000 revealed the presence of emphysematous bullae in the left upper lobe. In April of 2011, he experience increased sputum production and was referred to our hospital in November of the same year. A CT scan showed cavity formation and fluid accumulation in the left upper lobe. He was diagnosed as having CNPA based on the following laboratory results: Aspergillus fumigatus was cultured from hissputum, his $\beta$-D-glucan levels were elevated $(98.8 \mathrm{pg} / \mathrm{mL})$ and he was positive for the Aspergillus antibody. Despite treatment with the antifungal agents itraconazole, he developed a fever and was admitted to our hospital in January of 2012. His $\beta$-D-glucan level had increased to $3,210 \mathrm{pg} / \mathrm{mL}$. An anti-HIV-antibody test yielded negative results. Echocardiography revealed no cardiac vegetation. A. fumigatus was cultured from the sputum, but the blood culture results were negative. On hospital day 10, a contrastenhanced CT scan revealed a large, irregularly-shaped intraluminal filling defect in the aortic arch graft (Fig. 1). Based on the CT findings, we determined that the fungus ball was formed in the aorta by direct infiltration of Aspergillus. Because a similar structure was also seen at the bifurcation of the iliac arteries, dissemination of the fungus ball was speculated. In addition, an aneurysm was found in the

\footnotetext{
${ }^{1}$ Department of Respiratory Medicine, Saitama Cardiovascular and Respiratory Center, Japan, ${ }^{2}$ Division of Respiratory Diseases, Department of Internal Medicine, Jikei University School of Medicine, Japan and ${ }^{3}$ Department of Diagnostic Pathology, Saitama Cardiovascular and Respiratory Center, Japan

Received for publication January 23, 2013; Accepted for publication May 8, 2013

Correspondence to Dr. Hideaki Yamakawa, hide1144@jikei.ac.jp
} 


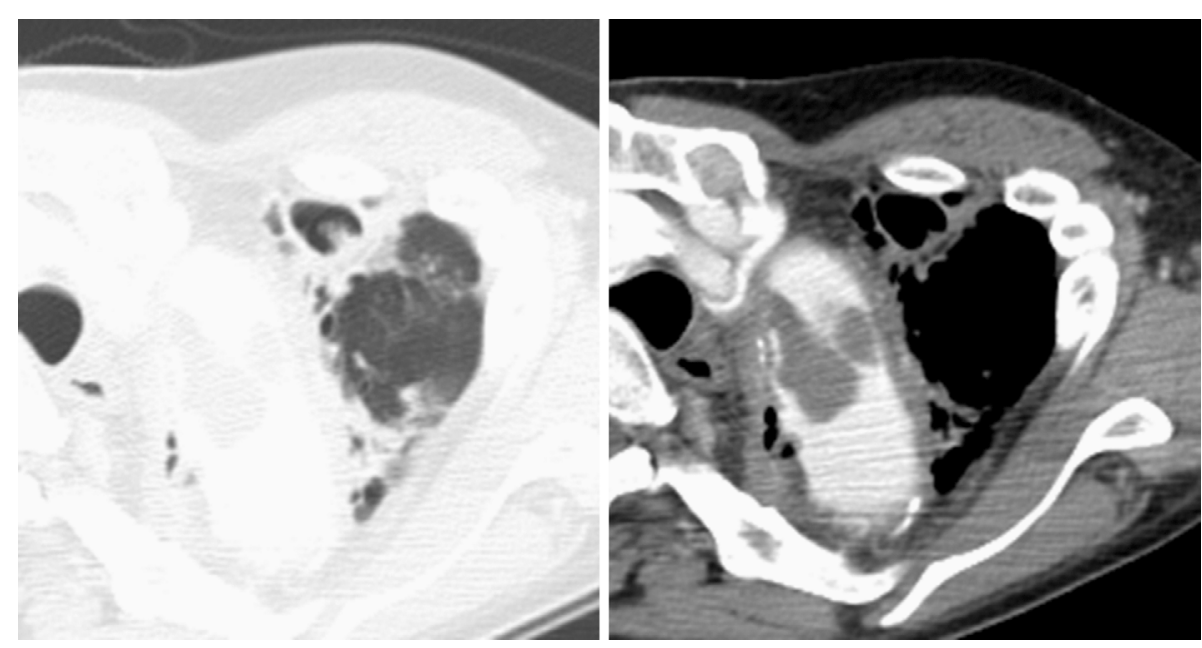

Figure 1. Chest contrast-enhanced computed tomography (CT) performed on hospital day 10. The chest contrast-enhanced CT scan revealed an irregularly shaped nonenhanced structure in the aortic arch graft.

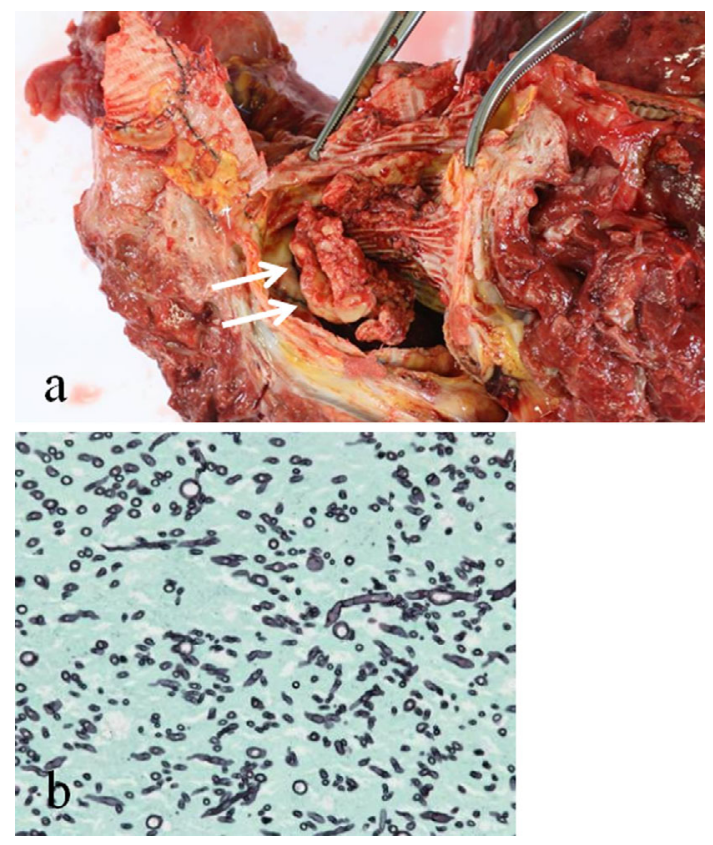

Figure 2. Macroscopic and microscopic histologic findings of the thoracic autopsy. (a) Fungus ball formation was revealed in the aortic arch graft (arrows). In addition, severe adhesions were found in the surrounding pleural and lung tissues. (b) Grocott staining of the tissues in the fungus ball region showed filamentous fungus with septa $(\times 200)$.

superior mesenteric artery. Although an antifungal regimin was administered including micafungin, liposomal amphotericin B and voriconazole, the treatment appeared to be ineffective. Surgical treatment was considered to be difficult because the entire thoracoabdominal aorta had been replaced by an artificial graft, and the site of infection was extensive. Despite the administration of additional antifungal therapies, the patient experience progressive respiratory failure, and he succumbed on hospital day 31 .

Autopsy findings revealed fungus ball formation in the aortic graft (Fig. 2), and A. fumigatus was cultured from this site. The formation of vegetation on the cardiac valve was not observed. The left lung section showed a convoluted cavity, and large numbers of A. fumigatus organisms were found upon the histological examination. Furthermore, sites of A. fumigatus infection were found in the wall of the superior mesenteric artery aneurysm and in the spleen. However, there was no invasion of A. fumigatus found between the pulmonary lesion and the aortic graft wall.

\section{Discussion}

This case demonstrated the formation of a fungus ball caused by an Aspergillus infection into an artificial aortic graft. The infection route was thought to be via either the direct infiltration of CNPA into the graft, an airborne infection during cardiac surgery or the infiltration of CNPA into the pulmonary vein followed by hematogenous dissemination to the graft. Regarding the first plausible cause, a case was reported in which CNPA directly infiltrated the aorta (1). However, the autopsy findings in this present case showed no infiltration of Aspergillus into the boundary region between the artificial graft and the pulmonary lesion. Therefore, this infection route was ruled out. Furthermore, there have been no reports of the direct infiltration of pulmonary aspergillosis into an artificial graft. Regarding the second plausible cause, an airborne infection route occurs most commonly between 5 weeks and 3 years postoperatively, and the diagnosis is made at 8 months on average. Because 7 postoperative years had passed in this case, it is unlikely that the disease was a surgical-related complication $(2,3)$. As a result, we believe that the third cause is likely, even though supportive histological findings could not be confirmed. This case showed disseminated aspergillosis with an infected aneurysm formation in the superior mesenteric artery and an infection site in the spleen.

The infiltration of Aspergillus into the blood, so-called in- 
vasive aspergillosis, generally develops only in immunodeficient patients. In this case, we could not elucidate a detailed mechanism of hematogenous infection by CNPA. To the best of our knowledge, cases with complications of CNPA associated with the infection of an artificial graft have not been reported.

Regarding treatment, Pasqualotto et al. reported a survival rate of only $7.3 \%$ out of 124 cases of endocarditis and aortitis caused by Aspergillus. In that study, surgical resection of the infected sites and the administration of antifungal agents were conducted in all patients (4).

Likewise, in the case of artificial graft infection, there are no reported cases of survival without the removal of the infected graft (2). Hanvesakul et al. reported that to improve the outcome of patients with this serious infection, early diagnosis, resection of as much of the infection site as possible and the administration of antifungal agents were vital to survival (5).

We herein reported the findings of an autopsy case of CNPA in which a fungus ball of A. fumigatus had formed in the thoracic aortic graft. Artificial aortic graft infection by Aspergillus could be one of the complications experienced by the patients with CNPA in addition to an aortic replacement by an artificial graft.

The authors state that they have no Conflict of Interest (COI).

\section{References}

1. Watanabe M, Hotchi M. An autopsy case of "fungus ball" type pulmonary aspergillosis complicated with direct invasion of thoracic aorta and systemic dissemination. Jpn J Med Mycol 27: 182189, 1986.

2. Doscher W, Krishnasastry KV, Deckoff SL. Fungal graft infections: case report and review of the literature. J Vasc Surg 6: 398402, 1987.

3. Aguado JM, Valle R, Arjona R, et al. Aortic bypass graft infection due to Aspergillus: report of a case and review. Clin Infect Dis 14: 916-921, 1992.

4. Pasqualotto AC, Denning DW. Post-operative aspergillosis. Clin Microbiol Infect 12: 1060-1076, 2006.

5. Hanvesakul R, Deshpande R, Carr CS, et al. Aspergillus aortitis: a cause for aortic perforation in a patient following combined aortic valve surgery and liver transplantation. Interact Cardiovasc Thorac Surg 3: 544-546, 2004.

(C) 2013 The Japanese Society of Internal Medicine http://www.naika.or.jp/imonline/index.html 